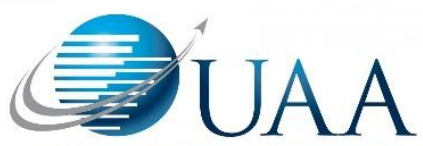

Collegiate Aviation Review

International

UNIVERSITY AVIATION

ASSOCIATION

\title{
The Relationship Between Pilot Attitudes and the Execution of Flight Safety Checklists
}

\author{
Barry H. Hyde \\ Federal Aviation Administration \\ David S. Cross \\ Embry-Riddle Aeronautical University
}

\begin{abstract}
A foundation of safe aircraft operation is correct checklist usage by the pilot. Correct checklist usage increases safe operations, which, in turn, creates a more efficient and economically-stable air transportation system. Pilots do not always properly execute their flight safety checklists before takeoff, during flight, and before landing airplanes, resulting in aviation accidents. The resultant business and social costs merit examination of the factors, attitudes, and training needed to minimize or eliminate accidents due to improper checklist execution. This quantitative research identified and evaluated the factors related to the improper execution of flight safety checklists with a survey of 109 certificated pilots regarding their usage and attitudes with respect to flight safety checklists. An important finding was that although no statistical difference was found, pilots with greater self-confidence and less risk orientation tended to place greater importance on the execution of flight safety checklists.
\end{abstract}

Recommended Citation:

Hyde, B.H., \& Cross, D.S. (2018). The Relationship Between Pilot Attitudes and the Execution of Flight Safety Checklists. Collegiate Aviation Review International, 36(2), 56-70. Retrieved from http://ojs.library.okstate.edu/osu/index.php/CARI/article/view/7407 
Overall accident rates in the aviation industry have declined since 1990 and changes in flight safety operations, combined with important advances in technology, have led to significant improvements in flight safety. The Federal Aviation Administration (FAA) has for more than two decades focused its training on pilot attitudes regarding aviation safety as a mechanism to prevent aviation accidents. However, little research has been done to assess the impact of pilot attitudes on behaviors cited as causal or contributing factors in aviation accidents. Pilots have trained to perform cockpit tasks by using checklist procedures during normal and abnormal operations. A checklist is a visual or oral aid used to identify and verify that items or steps were completed for a particular task, however, the improper performance of a flight safety checklist is a potential safety hazard. The improper performance of flight safety checklists for the purpose of this study includes skipping items on a checklist either intentionally or in error, or not executing a required checklist in its entirety. Improper checklist execution can occur due to pilot time constraints and because pilots forget to execute a checklist associated task after postponing it. Pilot performance of flight safety checklists is an integral part of flight safety as the checklists aid a pilot in completing tasks before takeoff, during flight, and before landing an airplane. Although the importance of flight safety checklists to safe flight has been well established, some pilots continue to take risks by not completely executing flight safety checklists. Risk is inherent to every flight, but how risk is perceived and influenced by pilot attitudes has implications for aviation safety.

\section{Background}

Flight safety checklists date back to the 1930s, predating the post-war interest and development of the human factors profession (Forsyth, 2007). During this time, checklists were developed because pilots were being injured and killed as a result of neglecting important tasks thought to be a result of relying solely on memory.

The complete execution of a flight safety checklist decreases the likelihood of an accident by removing the human factor of memory reliance. Rather, improper execution of a checklist occurs due to an improper attitude toward completion, pilot time constraints, and pilot failure to execute a checklist associated task after postponing it. Thus, any opportunity to improve checklist usage, such as through training, flight reviews, and pilot recertification should be considered to improve flight safety.

\section{Literature Review}

Previous research that examined the flight safety attitudes of pilots led Berlin et al. (1982) to develop a self-assessment instrument that was used to help identify hazardous pilot attitudes. The attitudes that were identified included the following: macho (characterized as a belief that one was better than others and risky behaviors that serve to justify this belief), antiauthoritarianism (characterized as a resentment of control by outside authority figures), impulsivity (characterized as a need to react quickly without thinking a situation through), resignation (characterized by a belief that one has no control over a situation), and invulnerability (characterized by a belief that one is immune to harm). 
In later research, the Aviation Safety Attitude Scale (ASAS) was developed to assess pilot attitudes regarding aviation safety issues with items reflecting the five hazardous attitudes in addition to items reflective of self-perceived skill and risk perception during flight. The ASAS included three pilot attitude subscales: self-confidence, or a pilot's confidence in his or her abilities related to operating an aircraft; risk orientation, the extent to which a pilot exhibited risky behavior; and safety orientation, the extent to which a pilot makes more cautious decisions. Research using ASAS suggested that risk perception was a key predictor of pilot behavior and was positively correlated with accident involvement (Hunter, 2005).

According to Parson (2008), aviation accidents associated with the improper execution of flight safety checklists occur because pilots do not completely execute their flight safety checklists before takeoff, during flight, or before landing. In this study the authors created a simple plan for dealing with emergency approaches. The plan included use of the ABCs (airspeed, best field for landing, checklist, etc.). This system, although generic, helped reinforce the criticality of checklists.

The Aircraft Owners and Pilots Association Air Safety Foundation (AOPAASF), the FAA, and the National Transportation Safety Board (NTSB) have identified a number of reasons for the improper execution of checklists. Some of these reasons include pilots unintentionally missing items or becoming complacent while checking important items on a flight safety checklist (AOPAASF, 2011; FAA, 2010; NTSB, 2012). Greater pilot compliance regarding checklist completion could lead to a reduction in aviation accidents and decreases in property damage and loss of life.

Risk tolerance is particularly important in safety-related professions, as workers are often exposed to workplace risks. The opportunity for pilots to engage in risky behavior is present daily. A pilot's risk tolerance can have a direct impact on safety (Ji, You, Lan, \& Yang, 2011). Pilots with high risk tolerance make decisions that expose them to unnecessary dangers, leading to increased chances of an accident. However, a more experienced pilot may consider a situation to be low risk; whereas, a less experienced pilot may consider this same situation to be high risk. For example, a pilot that is qualified to fly in instrument meteorological conditions may perceive less of a risk when encountering clouds and low visibility than a pilot who is only qualified to fly in visual meteorological conditions (Joseph \& Reddy, 2013). Underestimating the risk inherent in a particular situation and overestimating personal ability leads to a misperception of risk and these decision-making errors have been frequently found to contribute to aircraft accidents (Joseph \& Reddy, 2013).

Research has shown that hazardous pilot attitudes are important with respect to the relationships between risk perception, risk tolerance, and safety. These relationships are important because risk tolerance directly influences hazardous attitudes, which then directly influences safety (Ji et al., 2011). A pilot who does not execute a flight safety checklist exemplifies behavior directly related to a hazardous attitude associated with their risk perception. Pilots were trained to perform all checklist items and they know how important they are to safety. In some cases, however, pilots do not perceive that a flight safety checklist is necessary, thereby placing the flight at risk. A pilot's hazardous attitudes regarding safety increases their 
risk tolerance due to a number of factors, such as skipping items on a checklist due to time constraints, being interrupted and not returning to the stopping point on the checklist, and taking short cuts on the flight safety checklist because of fatigue.

Joseph and Reddy (2013) administered four questionnaires to a sample of 275 Indian Army helicopter pilots in order to study risk perception and safety attitudes regarding risky behavior and hazardous events. The four questionnaires consisted of: (1) the ASAS; (2) the Army Hazardous Event Scale (AHES), a 36-item scale used to assess a participant's involvement in hazardous aviation events; (3) the Risk Perception (Other) scale (RPO), a 17-item measure of a participant's perception of risk present in an aviation situation; and (4) the Risk Taking Tendency (RTT) scale, a five-item measure of a participant's risk-taking tendencies (Joseph \& Reddy, 2013). Results from the research revealed that high scores on risk-orientation, the extent to which a pilot exhibited risky behavior, and low scores on safety-orientation, the extent to which a pilot makes more cautious decisions, were related to a higher risk-taking tendency. The high risk-orientation and low safety-orientation scores were significantly associated with increased involvement in hazardous aviation events as measured by the ASAS (Joseph \& Reddy, 2013). Demonstration of the relationship between pilot attitudes, risk-taking tendency, and involvement in hazardous aviation events supported the use of the ASAS for aviation research studies pertaining to hazardous attitudes and safety related behaviors, such as the execution of flight safety checklists. The measures of safety attitudes, risk-perception, risk-taking, and involvement in hazardous aviation events could serve to assess precursors of accident risk among pilots (Joseph \& Reddy, 2013).

Several factors contributing to deviations in proper checklist execution have been identified. For example, pilots may rush through the execution of a flight safety checklist, thereby skipping items or become complacent with the execution of a flight safety checklist (FAA, 2010; NTSB, 2012). The National Aeronautics and Space Administration (NASA) analyzed human errors associated with the improper execution of flight safety checklists. NASA's project revealed five different patterns of human error associated with the improper execution of flight safety checklists, including: (1) reciting a flight safety checklist from memory and not actually executing the flight safety checklist; (2) the failure of a pilot to read a flight safety checklist to verify and crosscheck another pilot's actions, in cases where there was more than one pilot; (3) performing multiple checklist items together instead of individually; (4) early termination of or incomplete execution of a flight safety checklist; and (5) distractions leading to missed checklist items (Frakes \& Van Voorhis, 2007). In addition to different patterns of human error, errors related to the execution of flight safety checklists were generally classified into one of the following five categories: (1) checklist interruptions; (2) overlooking a checklist item; (3) using the wrong checklist; (4) checklist omission; and (5) checklist confusion (National Aeronautics and Space Administration, 2014). Checklist interruptions and distractions was the human error category that was most prevalent in ASRS reports involving checklist errors.

\section{Problem}

A pilot's attitudes affect his or her decision-making ability, thought patterns, and risk perceptions during all aspects of flight, which affects how he or she will respond to a given situation. Researchers have identified several specific hazardous pilot attitudes that are related to 
their willingness to violate regulations, exceed safety limits, or operate an aircraft in conditions beyond their capabilities (Berlin et al., 1982). The problem investigated was the potential devastating effects of not correctly completing flight safety checklists. Previous research suggests that improper execution of checklists has had devastating effects on pilots, property, and the air transportation system itself throughout aviation history.

\section{Purpose}

The purpose of this quantitative, correlational survey study was to examine whether a relationship exists between pilot attitudes and the execution of flight safety checklists. To examine the relationship between these variables, the ASAS was administered to pilots to assess their attitudes toward flight safety as well as the extent to which they properly executed all flight safety checklists. The theoretical perspective for this study is derived from the literature review, specifically, Berlin et. al. (1982) and Hunter (2005) whose research suggest that pilot attitudes and risky behavior influence safety behaviors. A better understanding of the effects of pilot attitudes will provide managers options for better processes and planning of aviation systems throughout the aviation industry.

\section{Research Question}

The research addressed the following research question:

Q: To what extent, if any, do the pilot attitudes self-confidence and risk orientation, as measured by the self-confidence and risk orientation subscales of the ASAS, respectively, predict the self-reported complete execution of all flight safety checklists for every flight, among participating pilots?

\section{Hypotheses}

H10: The pilot attitudes self-confidence and risk orientation, as measured by the selfconfidence and risk orientation subscales of the ASAS, respectively, do not significantly predict the self-reported complete execution of all flight safety checklists for every flight, among participating pilots.

H1a: The pilot attitudes self-confidence and risk orientation, as measured by the selfconfidence and risk orientation subscales of the ASAS, respectively, do significantly predict the self-reported complete execution of all flight safety checklists for every flight, among participating pilots.

\section{Research Method}

\section{Research Method and Design}

The research was conducted using a quantitative, correlational survey design to examine the relationship between hazardous pilot attitudes and the usage of flight safety checklists. A survey design was selected because the use of personal interviews did not allow for pilot 
anonymity. Participant anonymity was critical because it increased the likelihood of truthful participant responses. Pilots may be reluctant to report not executing a flight safety checklist without an assurance of anonymity.

The ASAS was selected for this study because it was developed to assess pilot attitudes regarding aviation safety issues with items reflecting the five hazardous attitudes in addition to items regarding the weather, risk perception, and self-perceived skill. The ASAS consists of 27 items, 10 of which reflect the five hazardous attitudes that were identified in previous research, including macho, antiauthoritarianism, impulsivity, resignation, and invulnerability (Hunter, 2005). The remaining 17 items assess pilot attitudes regarding the weather, risks encountered in aviation, the likelihood of experiencing an accident, and self-perceived skill. Exploratory principle component analysis of the ASAS identified three subscales representing selfconfidence, risk orientation, and safety orientation. For example, an item representing selfconfidence is "I am a very capable pilot." An example of a risk orientation item is "I would duck below minimums to get home." An example of a safety orientation item is "I am a very cautious pilot."

\section{Sample}

Pilots were recruited using the email newsletter distributed by the consulting firm Curt Lewis and Associates. The sample was one of convenience. A description of the proposed study and an invitation to participate in the research was provided within the email newsletter, which also included a link to the survey.

A power analysis was conducted using $\mathrm{G}^{*}$ Power (Faul, Erdfelder, Buchner, \& Lang, 2009) to determine the appropriate sample size for the multiple regression analysis using the following parameters: two predictor variables (self-confidence attitude and risk orientation attitude), a small effect size of .15, and an alpha and beta of .05 and .80 , respectively, resulting in a required sample size of 87 . A link to the survey was included as part of the newsletter.

\section{Materials and Instruments}

The ASAS consisted of 27 items that assessed pilot attitudes regarding aviation safety issues. Sample items from the scale included: I am a very careful pilot; I know aviation procedures very well; and, I deal with stress very well. A second section was included consisting of two items that assessed pilot attitudes regarding flight safety checklist usage: I believe flight safety checklists are necessary for every flight, and I completely execute all flight safety checklists for every flight. The sections utilized a Likert-type response scale that ranged from 5, strongly agree, to 1, strongly disagree. A third section included demographic questions assessing age, gender, and flight experience.

\section{Limitations}

One possible limitation to the proposed research is the use of participant self-reported data. Participants may be reluctant to respond honestly regarding whether they executed all flight safety checklists for all flights, given that the execution of all flight safety checklists was a 
requirement for every flight. One measure taken to mitigate this limitation was the assurance of participant anonymity.

\section{Findings}

This study included a survey of 109 pilots to examine the extent to which pilot attitudes of pilot self-confidence and risk orientation as measured by the self-confidence and risk orientation subscales of the Aviation Safety Attitude Scale (Hunter, 2005) predict the selfreported completion of all flight safety checklists for every flight among participating pilots.

A total of 109 responses were received from the study invitation. Of the 109 responses, $97(88.9 \%)$ study participants were male, and $12(11.1 \%)$ were female. The age distribution of participants included $3(2.8 \%)$ 18-25 years, 14 (12.8\%) 26-40 years, 57 (52.3\%) 41-59 years, 35 (32.1\%) 60 years or older. The majority, $70(64.2 \%)$ had more than 5,000 hours of total flight time. The most common type of pilot was airline transport $79(72.5 \%)$.

The ASAS scale measures three variables, self-confidence, risk orientation, and safety orientation. The researchers included three questionnaire items to measure pilot's execution of flight safety checklists.

Table 1 shows descriptive statistics for the independent variables, self-confidence and risk orientation regarding aviation safety. The self-confidence score had a possible range of 14 to 70. The mean, 52.9, was well above the midpoint of 42, indicating that on average, the study participants had a relatively high level of self-confidence regarding aviation safety. The risk orientation score had a possible range of 8 to 40. The mean, 16.7, was well below the midpoint of 24, indicating that on average, the study participants had a relatively low level of risk orientation (risk averse) regarding aviation safety.

Table 1

Descriptive Statistics for Aviation Safety Attitude Scores

\begin{tabular}{lccccc}
\hline & $N$ & & & & \\
\cline { 2 - 5 } & Valid & $M$ & $S D$ & Min & Max \\
\hline Self-confidence & 109 & 52.93 & 6.34 & 34.00 & 66.00 \\
Risk Orientation & 109 & 16.73 & 4.15 & 10.00 & 29.00 \\
\hline
\end{tabular}

Table 2 provides the frequency distributions for the two-flight safety checklist questionnaire items (dependent variables). Although every study participant answered either somewhat agree or strongly agree, these two responses are very different. That is, for maximum safety, all pilots should strongly agree to both questions. It could also be argued that those who only somewhat agreed have a less safe attitude regarding the use of flight safety checklists. Therefore, it is reasonable to compare the independent variables with these two dependent variables. 
Table 2

Frequency table for "I Believe Flight Safety Checklists are Necessary for Every Flight"

\begin{tabular}{lcc}
\hline & Frequency & Percent \\
\hline Somewhat Agree & 9 & 8.3 \\
Strongly Agree & 100 & 91.7 \\
Total & 109 & 100.0 \\
\hline
\end{tabular}

Frequency table for "I Completely Execute all Flight Safety Checklists for Every Flight"

\begin{tabular}{lcc}
\hline & Frequency & Percent \\
\hline Somewhat Agree & 22 & 27.2 \\
Strongly Agree & 59 & 72.8 \\
Total & 81 & 100.0 \\
\hline
\end{tabular}

The research question was analyzed in four areas:

H10: There is not a significant difference in the level of self-confidence regarding aviation safety between those who somewhat agreed and those who strongly agreed that flight safety checklists are necessary for every flight.

H20: There is not a significant difference in the level of risk orientation regarding aviation safety between those who somewhat agreed and those who strongly agreed that flight safety checklists are necessary for every flight.

H30: There is not a significant difference in the level of self-confidence regarding aviation safety between those who somewhat agreed and those who strongly agreed with the statement "I completely execute all flight safety checklists for every flight."

H40: There is not a significant difference in the level of risk orientation regarding aviation safety between those who somewhat agreed and those who strongly agreed with the statement "I completely execute all flight safety checklists for every flight." "RSQ1”).

Dependent Variable 1: Flight safety checklists are necessary for every flight (Labeled

Dependent Variable 2: I completely execute all flight safety checklists for every flight (Labeled "RSQ2").

Both questions were asked as Strong Agree (coded as "5") to Strongly Disagree (coded as "1"). Because the distribution of the both dependent variables was binary, logistic regression was determined appropriate for the analysis. Below, "5" = Strongly Agree and "4" = Agree" for both dependent variables. 


\section{Frequencies and Percentages}

The most frequently observed category of RSQ1 was $5(n=100,92 \%)$. The most frequently observed category of RSQ2 was $5(n=82,75 \%)$. Frequencies and percentages are presented in Table 3.

Table 3

Frequency Table for Nominal Variables

\begin{tabular}{lrr}
\hline Variable & $n$ & $\%$ \\
\hline RSQ1 & & \\
4 & 9 & 8.26 \\
5 & 100 & 91.74 \\
Missing & 0 & 0.00 \\
RSQ2 & & \\
4 & 27 & 24.77 \\
5 & 82 & 75.23 \\
Missing & 0 & 0.00 \\
\hline
\end{tabular}

It is important to note that the dependent variable for RSQ1 is less than 10, which means the regression analysis should be interpreted with caution given the rare-event data. However, given that there are two dependent variables, the overall results may be interpreted holistically.

Next, descriptive statistics were examined for the independent variables or predictors (Pilot Self Confidence and Pilot Risk Orientation). Both predictors are scales based on the Aviation Safety Attitude Scale (Hunter, 2005). Pilot Self Confidence were asked on Likert scale as Strong Agree (coded as "5") to Strongly Disagree (coded as "1").

\section{Summary Statistics}

The observations for Pilot_Self_Confidence had an average of $3.80\left(S D=0.32, S E_{M}=\right.$ 0.03, Min $=2.94$, Max = 4.44). The observations for Pilot_Risk_Orientation had an average of $2.23\left(S D=0.56, S E_{M}=0.05, \operatorname{Min}=1.14, \operatorname{Max}=4.14\right)$. Skewness and kurtosis were also calculated in Table 4 . When the skewness is greater than or equal to 2 or less than or equal to -2 , then the variable is considered to be asymmetrical about its mean. When the kurtosis is greater than or equal to 3, then the variable's distribution is markedly different than a normal distribution in its tendency to produce outliers (Westfall \& Henning, 2013). Both skewness and kurtosis fell in the normal range.

Table 4

Summary Statistics Table for Interval and Ratio Variables

\begin{tabular}{lrrrrrr}
\hline Variable & $M$ & $S D$ & $n$ & $S E_{M}$ & Skewness & Kurtosis \\
\hline Pilot_Self_Confidence & 3.80 & 0.32 & 109 & 0.03 & -0.41 & 0.08 \\
Pilot_Risk_Orientation & 2.23 & 0.56 & 109 & 0.05 & 0.91 & 1.03 \\
\hline
\end{tabular}




\section{Binary Logistic Regression}

Research Question 1 (RSQ1). A binary logistic regression was conducted to examine whether Pilot_Self_Confidence and Pilot_Risk_Orientation had a significant effect on the odds of observing the "5" category of RSQ1. The reference category for RSQ1 was a score of 4.

Assumptions. Variance Inflation Factors (VIFs) were calculated to detect the presence of multicollinearity between predictors. High VIFs indicate increased effects of multicollinearity in the model. Variance Inflation Factors greater than five are cause for concern, whereas VIFs of 10 should be considered the maximum upper limit (Menard, 2009). All predictors in the regression model have VIFs less than 10. Table 5 presents the VIF for each predictor in the model.

Table 5

Variance Inflation Factors for Pilot_Self_Confidence and Pilot_Risk_Orientation

\begin{tabular}{lr}
\hline Variable & VIF \\
\hline Pilot_Self_Confidence & 1.00 \\
Pilot_Risk_Orientation & 1.00 \\
\hline
\end{tabular}

Results. The overall model, $\chi^{2}(2)=0.49, p=.781$ was not significant, suggesting that Pilot_Self_Confidence and Pilot_Risk_Orientation did not have a significant effect on the odds of observing the 5 category of RSQ1. McFadden's R-squared was calculated to examine the model's fit, where values greater than .2 are indicative of models with excellent fit (Louviere, Hensher, \& Swait, 2000). The McFadden R-squared value calculated for this model was .01. Since the overall model was not significant, the individual predictors were not examined further. Table 6 summarizes the results of the regression model.

Table 6

Logistic Regression Results with Pilot_Self_Confidence and Pilot_Risk_Orientation Predicting RSQ1

\begin{tabular}{lrrrrr}
\hline Variable & $B$ & $S E$ & $\chi^{2}$ & $p$ & $O R$ \\
\hline (Intercept) & 4.22 & 4.37 & 0.94 & .333 & \\
Pilot_Self_Confidence & $(-0.24)$ & 1.08 & 0.05 & .826 & 0.79 \\
Pilot_Risk_Orientation & $(-0.40)$ & 0.58 & 0.48 & .487 & 0.67 \\
\hline
\end{tabular}

Note. $X^{2}(2)=0.49, p=.781, \mathrm{McFadden} R^{2}=0.01$.

Research Question 2 (RSQ2). Next, a binary logistic regression was conducted to examine whether Pilot_Self_Confidence and Pilot_Risk_Orientation had a significant effect on the odds of observing the "5" category of RSQ2. The reference category for RSQ2 was 4.

Assumptions. Variance Inflation Factors (VIFs) were calculated to detect the presence of multicollinearity between predictors. High VIFs indicate increased effects of multicollinearity in the model. Variance Inflation Factors greater than 5 are cause for concern, whereas VIFs of 10 should be considered the maximum upper limit (Menard, 2009). All predictors in the regression model have VIFs less than 10. Table 7 presents the VIF for each predictor in the model. 
Table 7

Variance Inflation Factors for Pilot_Self_Confidence and Pilot_Risk_Orientation

\begin{tabular}{lc}
\hline Variable & $V I F$ \\
\hline Pilot_Self_Confidence & 1.00 \\
Pilot_Risk_Orientation & 1.00 \\
\hline
\end{tabular}

Results. The overall model was not significant, $\chi^{2}(2)=0.09, p=.956$, suggesting that Pilot_Self_Confidence and Pilot_Risk_Orientation did not have a significant effect on the odds of observing the 5 category of RSQ2. McFadden's R-squared was calculated to examine the model's fit, where values greater than .2 are indicative of models with excellent fit (Louviere et al., 2000). The McFadden R-squared value calculated for this model was 0.00 . Since the overall model was not significant, the individual predictors were not examined further. Table 8 summarizes the results of the regression model.

Table 8

Logistic Regression Results with Pilot_Self_Confidence and Pilot_Risk_Orientation Predicting RSQ2

\begin{tabular}{lrrrrr}
\hline Variable & $B$ & $S E$ & $\chi^{2}$ & $p$ & $O R$ \\
\hline (Intercept) & 1.74 & 2.87 & 0.37 & .543 & \\
Pilot_Self_Confidence & $(-0.19)$ & 0.70 & 0.07 & .785 & 0.83 \\
Pilot_Risk_Orientation & 0.04 & 0.40 & 0.01 & .920 & 1.04 \\
\hline
\end{tabular}

Note. $X^{2}(2)=0.09, p=.956$, McFadden $R^{2}=0.00$.

\section{Discussion}

The intent of this research was to examine to what extent, if any, do the pilot attitudes self-confidence and risk orientation, as measured by the self-confidence and risk orientation subscales of the ASAS, respectively, predict the self-reported complete execution of all flight safety checklists for every flight, among participating pilots.

H10: There is not a significant difference in the level of self-confidence regarding aviation safety between those who somewhat agreed and those who strongly agreed that flight safety checklists are necessary for every flight.

The results indicated some evidence to suggest that pilots who strongly agreed that flight safety checklists are necessary for every flight tend to have more self-confidence regarding aviation safety compared to those who only somewhat agreed that flight safety checklists are necessary for every flight. However, there was not a statistically significant difference between the two groups. The null hypothesis was not rejected and it was concluded that there is insufficient evidence to suggest there is a difference in the level of self-confidence in aviation safety between those who somewhat agreed and those who strongly agreed with the statement. 
H20: There is not a significant difference in the level of risk orientation regarding aviation safety between those who somewhat agreed and those who strongly agreed that flight safety checklists are necessary for every flight.

The results indicated some evidence to suggest that pilots who strongly agreed that flight safety checklists are necessary for every flight tend to have less risk orientation regarding aviation safety compared to those who only somewhat agreed that flight safety checklists are necessary for every flight. However, there was not a statistically significant difference between the two groups. The null hypothesis was not rejected and it was concluded that there is insufficient evidence to suggest that pilots who strongly agreed that flight safety checklists are necessary for every flight tend to have less risk orientation regarding aviation safety compared to pilots who only somewhat agreed with the statement.

H30: There is not a significant difference in the level of self-confidence regarding aviation safety between those who somewhat agreed and those who strongly agreed with the statement "I completely execute all flight safety checklists for every flight."

The results indicated some evidence to suggest that pilots who strongly agreed with the statement "I completely execute all flight safety checklists for every flight" tend to have greater self-confidence regarding aviation safety compared to those who only somewhat agreed with the statement. However, there was not a statistically significant difference between the two groups. The null hypothesis was not rejected and it was concluded that there is insufficient evidence to suggest that pilots who strongly agreed with the statement "I completely execute all flight safety checklists for every flight" tend to have greater self-confidence regarding aviation safety compared to those who only somewhat agreed with the statement.

H40: There is not a significant difference in the level of risk orientation regarding aviation safety between those who somewhat agreed and those who strongly agreed with the statement "I completely execute all flight safety checklists for every flight."

The results indicated some evidence to suggest that pilots who strongly agreed with the statement "I completely execute all flight safety checklists for every flight" tend to have a lower level of risk orientation regarding aviation safety compared to those who only somewhat agreed with the statement. However, there was not a statistically significant difference between the two groups. The null hypothesis was not rejected and it was concluded that there is insufficient evidence to suggest that pilots who strongly agreed with the statement "I completely execute all flight safety checklists for every flight" tend to have less risk orientation regarding aviation safety compared to those who only somewhat agreed with the statement.

The findings indicated that a study with a larger number of participants and more discriminatory questions is needed to determine whether there is a relationship between pilot attitudes and their self-reported use of flight safety checklists. Analysis of the demographic data revealed that the sample was not representative of the entire pilot population because of the high number of pilots with airline transport pilot qualifications and flight instructor certificates. It is likely that these more experienced pilots would have more positive habits and attitudes associated with flight safety checklists. A solution may be to exclude airline transport pilots from 
a future study, thereby focusing on general aviation pilots exclusively. Another solution would be to solicit feedback from private pilots that fly for pleasure and eliminate any commercial pilots. It would also be important in future research to provide the questionnaire to a wider variety of pilots as this questionnaire was available only to pilots who used this one website and may not be representative of the total pilot population. Additional items should also be added to gather more specific information on the quality of checklist usage versus whether or not a checklist was completed. These items could include whether checklists are read out loud, whether they are restarted when interrupted, and whether their initiation is associated with salient events. This study is a first step in evaluating whether an emphasis on pilot attitudes in flight training is a meaningful way to affect pilot behavior with respect to issues of flight safety, such as the use of checklists.

\section{Conclusions}

In conclusion, these research findings may not be generalizable to the entire population of general aviation pilots because of limited sample size and the convenience sampling method used. Even with these limitations, an important finding was that pilots vary in the extent to which they regard the necessity and importance of flight safety checklists. The data suggest that checklist usage, which is a fundamental part of aviation safety practices from the very first flight lesson, may not be considered at the critical level of importance that it needs to be to achieve the greatest safety benefits. If flight safety checklists are not always regarded at the highest level of importance by a pilot for every flight, this may be an indication that there is a need to reemphasize training on this particular skill more effectively.

\section{Recommendations}

Based on the findings from this study, one recommendation is that pilot training should continue to focus on the importance of executing all flight safety checklists for every flight. Pilots in this study all agreed that checklists are necessary for every flight, however, some of the pilots only somewhat agreed with this statement indicating there is opportunity for improvement.

Although there were indications that self-confidence and risk orientation affected how strongly pilots regarded checklist usage, no strong conclusions could be made that the focus of training on pilot attitudes would affect checklist usage. To ensure the proper execution of checklists, pilot training needs to focus on the proper and consistent execution of checklists and not solely focus on attitudes which affect behavior. Training should focus on those factors that have been shown to affect the proper deliberate execution of checklists rather than performing checklists in a mechanical fashion that is vulnerable to errors. If pilots do not strongly regard the execution of checklists, then it may be that the pilots are not performing the checklists properly or as consistently as they should be, indicating that more training on this behavior is warranted by the findings.

\section{Future Research}

Significant value can be derived from this research for the additional future research that can build upon these initial findings. Future research needs to focus on private pilots from a 
wider demographic area by soliciting through multiple means. A larger sample would broaden the scope of future research. More research should also focus more on the quality of the checklist execution process versus the importance of checklist execution completion. It is important that future research recognizes the trends in checklist usage that is redirecting general aviation away from the use of manual checklists and toward the use of electronic checklists. Flight safety attitudes identified in previous research by Berlin et al. (1982) could also be used comparatively with checklist usage in future research. In addition, future research should take into account a larger level of detail related to Hunter (2005) including pilot experience, aircraft complexity, phase of flight, and other key variables that may influence both human factors and checklist significance. 


\section{References}

Aircraft Owners and Pilots Association Air Safety Foundation. (2011). Joseph T. Nall report, accident trends and factors for 2010. Retrieved from https://www.aopa.org//media/files/aopa/home/training-and-safety/nall-report/11nall.pdf

Berlin, J. I., Gruber, E. V., Holmes, C. W., Jensen, P. K., Lau, J. R., Mills, J. W., \& O’Kane, J. M. (1982). Pilot judgment training and evaluation (Volume I) (DOT/FAA/CT-82/56-I). Retrieved from http://www.tc.faa.gov/ its/worldpac/techrpt/ct82-56-1.pdf

Faul, F., Erdfelder, E., Buchner, A., \& Lang, A. G. (2009). Statistical power analyses using G*Power 3.1: Tests for correlation and regression analyses. Behavior Research Methods, 41, 1149-1160.

Federal Aviation Administration. (2010). Abbreviations, acronyms and definitions. Retrieved from https://www.faa.gov/jobs/abbreviations/

Forsyth, E. (2007). Human factors in aviation/aerospace industry. Daytona Beach, FL: EmbryRiddle Aeronautical University.

Frakes, M., \& Van Voorhis, S. (2007). Effectiveness of a challenge-and-respond checklist in ensuring safety behavior compliance by medical team members at a rotor-wing air medical program. Air Medical Journal, 26(5), 248-251. doi:10.1016/j.amj.2007.05.009

Hunter, D. (2005). Measurement of hazardous attitudes among pilots. International Journal of Aviation Psychology, 15(1), 23-43. https://doi.org/10.1207/s15327108ijap1501_2

Ji, M., You, X., Lan, J., \& Yang, S. (2011). The impact of risk tolerance, risk perception and hazardous attitude on safety operation among airline pilots in China. Safety Science, 49, 1412-1420. https://doi.org/10.1016/j.ssci.2011.06.007

Joseph, C., \& Reddy, S. (2013). Risk perception and safety attitudes in Indian army aviators. International Journal of Aviation Psychology, 23(1), 49-62. https://doi.org/10.1080/ 10508414.2013.746531

Louviere, J. J., Hensher, D. A., \& Swait, J. D. (2000). Stated choice methods: Analysis and applications. Cambridge University Press. Cambridge, UK.

Menard, S. (2009). Logistic regression: From introductory to advanced concepts and applications. Sage Publications. Thousand Oaks, CA.

National Aeronautics and Space Administration. (2014). Aviation safety reporting system: Callback. Retrieved from http://asrs.arc.nasa.gov

National Transportation Safety Board. (2012). Improve general aviation safety. NTSB most wanted list. Retrieved from www.ntsb.gov/safety/mw15_2012.html 
Hyde \& Cross: The Relationship Between Pilot Attitudes and the Execution of Flight Safety Checklists

Parson, S. (2008). ABCDE emergencies step by step: Teach emergencies with the ABCs. FAA Safety Briefing. Retrieved from www.faa.gov/news/safety_briefing/2008/media/ mayjun2008.pdf

Westfall, P. H., \& Henning, K. S. S. (2013). Texts in statistical science: Understanding advanced statistical methods. Boca Raton, FL: Taylor \& Francis. 